\title{
Effects of carbamazepine combined with vitamin B12 on levels of plasma homocysteine, hs-CRP and TNF- $\alpha$ in patients with epilepsy
}

\author{
TIANCHI ZHOU ${ }^{1}$, NUAN WANG ${ }^{2}$, LEI XU ${ }^{3}$, HONGLI HUANG $^{2}$, CHUNYU YU $^{2}$ and HAO ZHOU ${ }^{2}$ \\ ${ }^{1}$ School of Clinical Medicine, Kangda College of Nanjing Medical University, Lianyungang, Jiangsu 222002; \\ Departments of ${ }^{2}$ Neurology and ${ }^{3}$ Pharmacy, The First People's Hospital of Xuzhou, Xuzhou, Jiangsu 221000, P.R. China
}

Received August 18, 2017; Accepted December 11, 2017

DOI: $10.3892 /$ etm.2018.5698

\begin{abstract}
The efficacy of carbamazepine combined with vitamin B12 in epilepsy treatment by comparing levels of plasma homocysteine (Hcy), serum TNF- $\alpha$ and hs-CRP in patients with epilepsy before and after treatment was investigated. Fifty-eight patients with epilepsy who were admitted and received treatment in The First People's Hospital of Xuzhou were recruited as subjects, and fifty-eight healthy volunteers were recruited as the control group. Patients were treated with carbamazepine combined with vitamin B12 for a period of three months. The mRNA and protein levels of TNF- $\alpha$ and hs-CRP in serum were measured before and after treatment using semi-quantitative RT-PCR and western blotting, respectively. The plasma Hcy levels were measured as well. Within one year after the 3-month treatment, the frequency and duration of seizure were tracked. After treatment with carbamazepine combined with vitamin B12 for patients with epilepsy, the Hcy level was significantly higher than that before treatment and that in the control group $(\mathrm{P}<0.01)$. The mRNA and protein levels of TNF- $\alpha$ and hs-CRP in serum were significantly higher in patients than that in healthy people $(\mathrm{P}<0.01)$. After treatment these levels were reduced $(\mathrm{P}<0.01)$, but still higher than those in healthy people $(\mathrm{P}<0.05, \mathrm{P}<0.01)$. After treatment, the frequency and duration of seizures were all reduced $(\mathrm{P}<0.05, \mathrm{P}<0.01)$. The results suggested that carbamazepine combined with vitamin B12 was effective in treatment of epilepsy by reducing levels of TNF- $\alpha$ and hs-CRP in the serum, but had a risk of increasing the Hcy level.
\end{abstract}

\section{Introduction}

Epilepsy, as one of the common neurological disorders, is actually a paroxysmal disorder mainly caused by abnormal

Correspondence to: Dr Hao Zhou, Department of Neurology, The First People's Hospital of Xuzhou, 19 Zhongshanbei Road, Xuzhou, Jiangsu 221000, P.R. China

E-mail: hao_zhou9@163.com

Key words: epilepsy, carbamazepine, homocysteine, TNF- $\alpha$ discharge or synchronization of brain neurons $(1,2)$. Seizures can take on many different forms and occur at any time. The incidence of epilepsy is only lower than that of stroke. Refractory epilepsy has a high mortality rate, imposing a great burden on the family and society (3). A single antiepileptic drug is generally prescribed for treating epilepsy. At present, there are various kinds of antiepileptic drugs available clinically for treatment of complex epilepsy. However, drug resistance occurs often in patients for antiepileptic drugs. When patients respond poorly to monotherapy, combination therapies are likely to be used for the treatment of seizures (4). Due to the fact that carbamazepine is a first-line drug currently for treatment of epilepsy, there is a high risk that patients will acquirer drug resistance (5). It was reported that antiepileptic drugs combined with vitamin supplements reduced the drug resistance that patients experienced, thus increasing the cure rate of refractory epilepsy (6). In another study, antiepileptic drugs were found to be able to increase the level of plasma homocysteine (Hcy), which is one of the major causes leading to cardiovascular disease. The levels of serum high-sensitivity C-reactive protein (hs-CRP) and tumor necrosis factor (TNF- $\alpha$ ) were found to be increased as well (7). Of note, Hermann et al (8) found that even without drug intervention the serum levels of hs-CRP and TNF- $\alpha$ in patients with epilepsy were significantly higher than normal levels in healthy people.

In this study, the effects of carbamazepine combined with vitamin B12 on levels of plasma homocysteine, serum hs-CRP and TNF- $\alpha$ in patients with epilepsy were explored aiming to provide new ideas for clinical treatment of epilepsy.

\section{Patients and methods}

Patients. A total of 58 patients were recruited who were admitted to the Neurology Department from February 2014 to February 2015 and were diagnosed with epilepsy based on clinical examination and the Operational Classification of Seizure Types by the International League Against Epilepsy. The patients were aged 29-53 years, consisting of 27 males and 31 females. All enrolled patients were with primary epilepsy for an average of $13.27 \pm 5.52$ years, and experienced an average of $7.18 \pm 2.86$ seizures per year. All other consumptive diseases were excluded. An informed consent form was signed by all 
patients or their family members. This study was approved by the Ethics Committee of the First People's Hospital of Xuzhou. Fifty-eight healthy volunteers were recruited as the control group. There were no statistically significant differences in age and gender between the recruited patients and volunteers. All of these patients had complete surgical data and complete treatment regimen.

Instruments and materials. The following materials and instruments were purchased from commercial vendors: carbamazepine tablets from Novartis (CFDA approval no. H11022279; Shanghai, China); vitamin B12 tablets from Guangdong Yixiang Pharmaceuticals (CFDA approval no. H44020621; Guangdong, China); TNF- $\alpha$ ELISA kit, hs-CRP ELISA kit, TRIzol kit, reverse transcrip-tion kit, and ECL reagent from Invitrogen (Carlsbad, CA, USA); human homocysteine assay kit from B\&D Systems (Bedford, MA, USA); primary rabbit anti-human TNF- $\alpha$, hs-CRP, and gAPDH polyclonal antibodies (1:1,000; cat. nos. 6945, 14316 and 2118), and horseradish peroxidase conjugated mouse anti-rabbit secondary polyclonalantibody $(1: 2,000$; cat no. 93702) from Cell Signaling Technology (Beverly, MA, USA); PCR instrument from Applied Biosystems (Foster, CA, USA); UV imaging system and electronic balance (BP121S) from Biometra (Goettingen, Germany). Other instruments and reagents are described in relevant context.

Treatment methods and sample collection. After diagnosis and before any treatment, a sample of whole blood was drawn from all patients and volunteers in a fasting and resting state. The blood sample was centrifuged at 2,010 $\mathrm{x}$ g for $20 \mathrm{~min}$ to separate serum and plasma. The isolated serum and plasma samples were stored at $-80^{\circ} \mathrm{C}$ for future use. Then treatment started. The patients were given orally carbamazepine at a dose of $200 \mathrm{mg}$ per day combined with vitamin B12 at a dose of $50 \mu \mathrm{g}$ per day for a period of three months. During the treatment, the frequency and severity of seizures were closely monitored. During this time, the volunteers in the control group received no treatment. After the three-month treatment, another sample of whole blood was drawn from the patients in a fasting and resting state. Serum and plasma were isolated and stored at $-80^{\circ} \mathrm{C}$.

Measurement of plasma Hcy levels. The levels of plasma Hcy in each group were measured by enzymatic cycling assay. A Hcy assay kit was used in the measurement, and the manual included in the kit was closely followed. A standard curve was first established using Hcy standard solutions with different concentration.

Then 5 samples with known concentration were measured eight times to verify the accuracy of the method. The plasma samples were diluted 50 times with diluent before assay, and the Hcy concentration was calculated by using the standard curve.

Determination of TNF- $\alpha$ and hs-CRP levels in serum by ELISA. The ELISA kit manual was followed. A concentrated stock solution was made for TNF- $\alpha$ and hs-CRP, respectively. Standard solutions were prepared by serial dilution of the stock solution with Diluent buffer for generating a standard curve.
Table I. PCR primer sequences.

\begin{tabular}{ll}
\hline Gene name & \multicolumn{1}{c}{ Primer sequences } \\
\hline TNF- $\alpha$ & F: 5'-CAGCCTCTTCTCCTTCCTGA-3' \\
& R: 5'-GGAAGACCCCCCCAGATAGA-3' \\
hs-CRP & F: 5'-TCGAGGTTCCTGAAGTCACA-3' \\
& R: 5'-AACAAAGGCCCAGAGACAGA-3' \\
GAPDH & F: 5'-TGGGTGTGAACCATGAGAA-3' \\
& R: 5'-GGCATGGACTGTGGTCATGA-3'
\end{tabular}

$\mathrm{F}$, forward; $\mathrm{R}$, reverse.

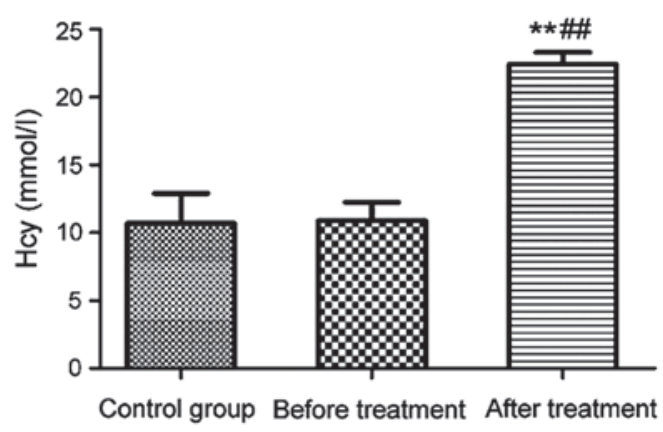

Figure 1. Plasma Hcy levels of the control group and patients with epilepsy before and after treatment. ${ }^{* *} \mathrm{P}<0.01$, compared with the control group; ${ }^{\# \#} \mathrm{P}<0.01$, compared with patients before treatment.

The biotin-labeled human TNF- $\alpha$ and hs-CRP antibodies were diluted and mixed. The wells of an ELISA plate were added with the prepared standards or samples. The plate was covered with a film and incubated at $37^{\circ} \mathrm{C}$ for $90 \mathrm{~min}$. The liquid in the plate was then removed and the wash buffer added. After 3 washes, the avidin-peroxidase complex was added, and the plate was covered with a film and incubated at $37^{\circ} \mathrm{C}$ for $30 \mathrm{~min}$. The liquid was discarded, and chromogenic substance solution was added, and the plate was covered with a film and incubated at $37^{\circ} \mathrm{C}$ in the dark for $30 \mathrm{~min}$. A stop solution was added to each well to quench the reaction. The solution in the wells turned from blue to yellow. The optical density (OD) value of each well was measured at $480 \mathrm{~nm}$ with a microplate reader, and compared to the standard curve to obtain the levels of TNF- $\alpha$ and hs-CRP in serum.

Expression levels of TNF- $\alpha$ and hs-CRP mRNA measured by semi-quantitative $P C R$. The serum samples stored at $-80^{\circ} \mathrm{C}$ were thawed, and followed by adding same volume of TRIzol reagent. The protocol in the manual of the RNA extraction kit was followed to extract the total RNA in the serum samples. The concentration and purity of the extracted RNA were determined by UV spectrophotometer. Generally the A260/A280 ratio was between 1.8 and 2.0 for the extracted RNA. The RNA integrity was assessed by $2 \%$ agarose gel electrophoresis analysis. The $28 \mathrm{~S}, 18 \mathrm{~S}$ and $5 \mathrm{~S}$ bands were clearly visible in the electrophoretogram, and the luminance of the $28 \mathrm{~S}$ band was about twice that of $18 \mathrm{~S}$. This gel assessment result indicated that the extracted RNA was intact and 

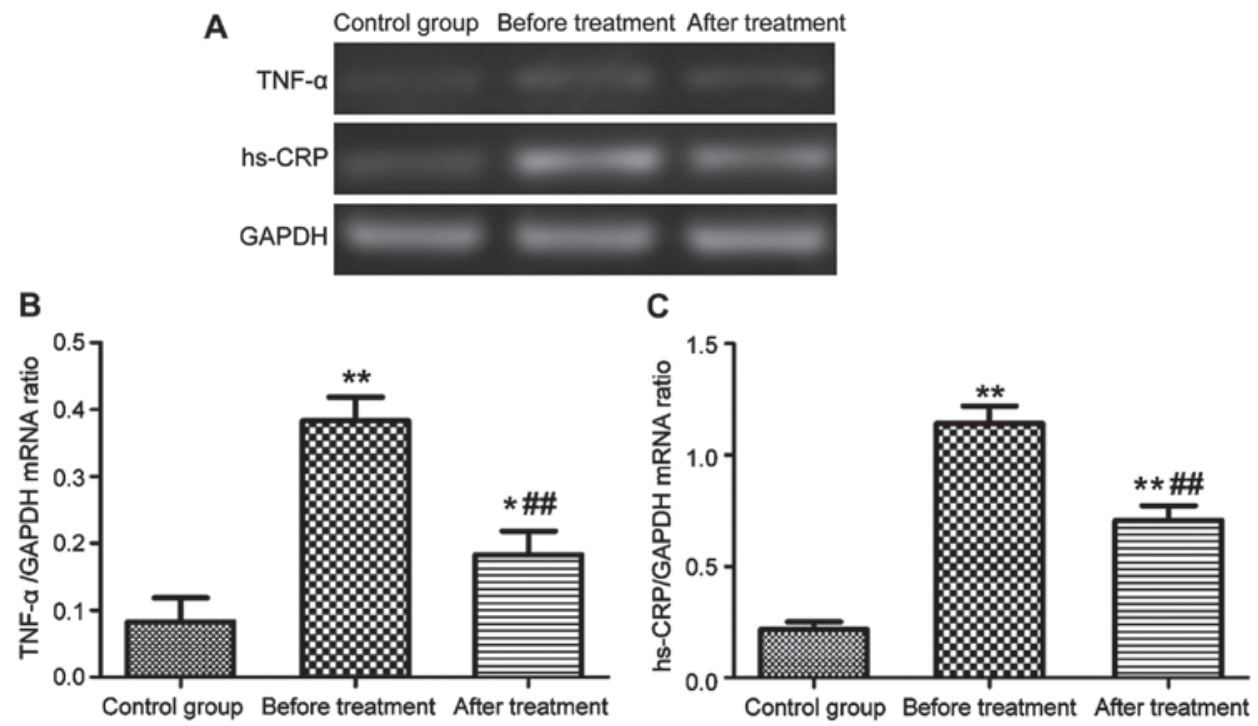

Figure 2. Serum levels of TNF- $\alpha$ and hs-CRP mRNA. (A) Gel electrophoresis map; (B and C) bar graphs of TNF- $\alpha$ and hs-CRP mRNA expression levels, respectively $\left({ }^{*} \mathrm{P}<0.05,{ }^{* *} \mathrm{P}<0.01,{ }^{\# \#} \mathrm{P}<0.01\right)$.

can be used for subsequent experiments. All the devices used to extract RNA in this experiment were RNase-free. cDNA was synthesized from the extracted RNA via reverse transcription using a reverse transcription kit. The reaction conditions were $95^{\circ} \mathrm{C}$ for $1 \mathrm{~min}, 65^{\circ} \mathrm{C}$ for $5 \mathrm{~min}$, and $56^{\circ} \mathrm{C}$ for $15 \mathrm{~min}$. The obtained cDNA samples were stored at $-20^{\circ} \mathrm{C}$ for later use. The expression levels of TNF- $\alpha$ and hs-CRP mRNA were measured by semi-quantitative PCR using GAPDH as internal reference. The primers listed in Table I were synthesized by Tiangen Biotech Co., Ltd and were used in the PCR amplification. PCR conditions were as follows: $95^{\circ} \mathrm{C}$ for $5 \mathrm{~min}$; then 40 cycles of $95^{\circ} \mathrm{C}$ for $45 \mathrm{sec}, 58^{\circ} \mathrm{C}$ for $35 \mathrm{sec}$ and $72^{\circ} \mathrm{C}$ for $60 \mathrm{sec}$; and finally $72^{\circ} \mathrm{C}$ for $5 \mathrm{~min}$. The PCR products were then analyzed with agarose gel electrophoresis. The intensity of the bands was analyzed by a UV imaging system. The expression levels of TNF- $\alpha$ and hs-CRP mRNA in the serum samples were represented by ratios of TNF- $\alpha / \mathrm{GAPDH}$ and hs-CRP/GAPDH.

Expression levels of TNF- $\alpha$ and hs-CRP determined by western blotting. Serum samples were centrifuged at $10,800 \mathrm{x} g$ for 10 min at $4^{\circ} \mathrm{C}$, and the supernatant was collected. The total protein concentration was measured using a BCA protein quantification kit (Invitrogen). Equal amount of protein from different samples was taken to prepare the sample system, which was boiled for $15 \mathrm{~min}$ to denature the protein before separation by $15 \%$ SDS-PAGE. After the electrophoresis, the protein bands were transferred from gel to PVDF membrane by wet transfer method. The membrane was then washed with TBST to remove residual transfer buffer, and blocked for $2 \mathrm{~h}$ in 5\% skim milk powder. After blocking, the target protein bands were cut and incubated in primary antibodies of TNF- $\alpha$, hs-CRP and GAPDH (1:1,000 dilution) overnight at $4^{\circ} \mathrm{C}$. Following 3 washes with TBST for 5 min each, the bands were incubated in the secondary antibody (1:5000 dilution) at room temperature for $2 \mathrm{~h}$. After 3 washes with TBST, the membrane was incubated in appropriate amount of ECL reagent (1:1 of reagent A and B) in the dark. A film was placed against the blot membrane and exposed for an appropriate time period depending on the fluorescence intensity of the blot. The exposed film was then developed and fixed. The blot was scanned and analyzed using ImageJ software for gray value analysis. The expression levels of TNF- $\alpha$ and hs-CRP were analyzed statistically using gray value ratios of TNF- $\alpha /$ GAPDH and hs-CRP/GAPDH.

Prognosis assessment. A comprehensive evaluation of prognosis was undertaken based on the EEG index and clinical symptoms before and after treatment. The efficacy of carbamazepine combined with vitamin B12 on epilepsy treatment was evaluated according to improvement in epileptic symptoms including the frequency and duration of seizures after treatment.

Statistical analysis. The data in this study were expressed as mean \pm standard deviation. The SPSS 19.0 software was used for data processing (SPSS Inc., Chicago, IL, USA). Variance analysis was performed for comparison between groups. Homogeneity of variance was tested. If the homogeneity of variance assumption held, then the Bonferroni correction was used for pairwise comparison. If the homogeneity of variance assumption was violated, then the Welch test was used. Dunnett's T3 test was used for multiple comparisons. $\mathrm{P}<0.05$ was considered to indicate a statistically significant difference.

\section{Results}

Plasma Hcy levels of patients with epilepsy. The levels of plasma Hcy were measured by enzymatic cycling assay for patients before and after treatment. The result in Fig. 1 showed that the plasma Hcy level of patients before treatment was comparable to that of the control group ( $\mathrm{P}>0.05)$. After 3 months of treatment with carbamazepine combined with vitamin B12, the plasma Hcy level of patients was significantly higher than those of the control group and patients before treatment $(\mathrm{P}<0.01)$. 
A

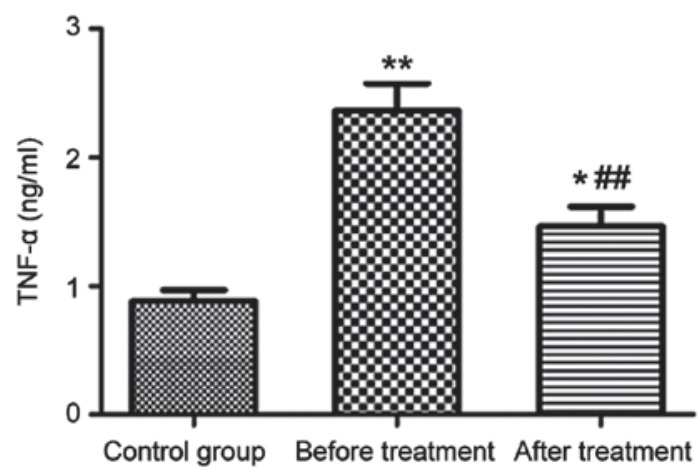

B

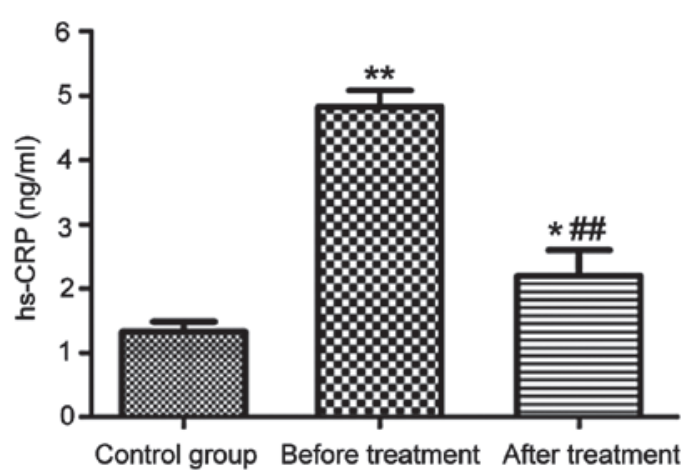

Figure 3. Levels of TNF- $\alpha$ (A) and hs-CRP (B) in serum measured by ELISA ${ }^{*} \mathrm{P}<0.05,{ }^{* *} \mathrm{P}<0.01,{ }^{\# \#} \mathrm{P}<0.01$.

A

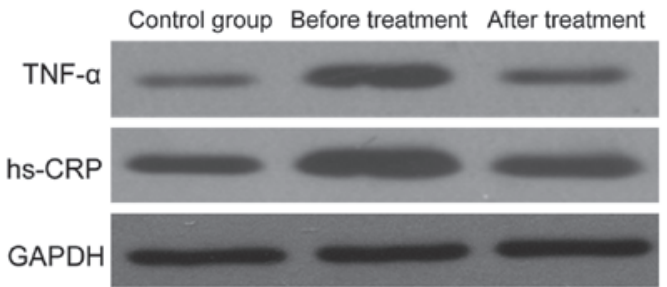

B

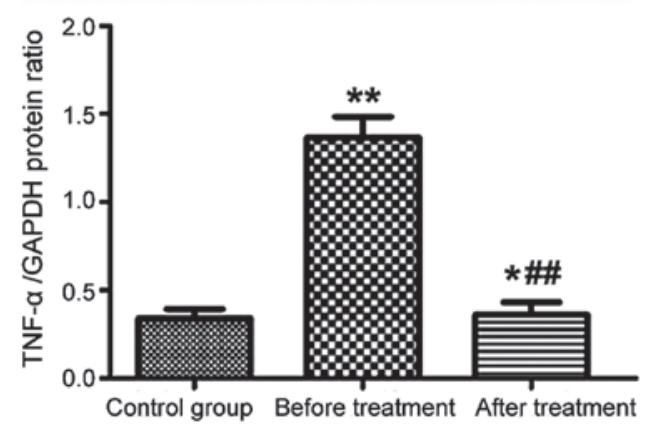

C

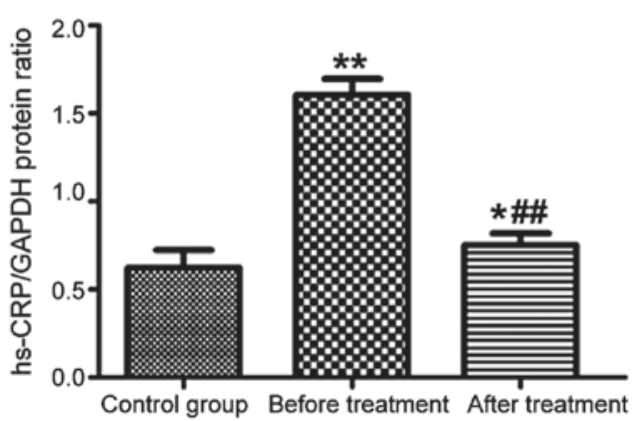

Figure 4. Levels of TNF- $\alpha$ and hs-CRP protein in serum measured by western blotting (A). (B and C) Bar graphs of TNF- $\alpha$ and hs-CRP protein expression levels, respectively $\left({ }^{*} \mathrm{P}<0.05,{ }^{* * *} \mathrm{P}<0.01,{ }^{, \#} \mathrm{P}<0.01\right)$.

Serum expression levels of TNF- $\alpha$ and hs-CRP mRNA in patients with epilepsy. The levels of TNF- $\alpha$ and hs-CRP mRNA in serum were measured by semi-quantitative RT-PCR. As shown in Fig. 2, the serum levels of TNF- $\alpha$ and hs-CRP mRNA in patients with epilepsy were significantly higher than those in the control group $(\mathrm{P}<0.01)$. After 3 months of treatment with carbamazepine combined with vitamin B12, the serum levels of TNF- $\alpha$ and hs-CRP mRNA were significantly reduced $(\mathrm{P}<0.01)$, but still much higher than those in the control group $(\mathrm{P}<0.01, \mathrm{P}<0.05)$.

Serum levels of TNF- $\alpha$ and hs-CRP in patients with epilepsy. The levels of TNF- $\alpha$ and hs-CRP in serum were measured by both ELISA and western blotting. The two detection methods gave consistent results, as shown in Fig. 3 (ELISA) and Fig. 4 (western blotting). The serum levels of TNF- $\alpha$ and hs-CRP in patients with epilepsy were significantly higher than those in the control group $(\mathrm{P}<0.01)$. After 3 months of treatment with carbamazepine combined with vitamin B12, the levels of TNF- $\alpha$ and hs-CRP in serum were significantly reduced $(\mathrm{P}<0.01)$, but still higher than those in the control group $(\mathrm{P}<0.05)$.
Seizure frequency and duration of epilepsy after treatment. Patients with epilepsy were followed up for 1 year after treatment with carbamazepine combined with vitamin B12 for 3 months. Seizure frequency and duration were tracked and compared with those before treatment. As shown in Fig. 5, the frequency of seizures was significantly reduced after treatment $(\mathrm{P}<0.01)$, and the duration of each seizure was shortened as well $(\mathrm{P}<0.05)$.

\section{Discussion}

Epilepsy is characterized by abnormal electrical discharge from the brain due to complex causes, making the neurons over-excited. Patients have frequent recurrent seizures, and may experience loss of control of their emotion, physical feelings and consciousness temporarily during a seizure. Epilepsy can occur to people at any age $(9,10)$. Carbamazepine, as a first line antiepileptic drug, is a sodium channel blocker. It reduces sodium channel permeability to sodium, and thus decreases cellular excitability and extends the refractory period. Carbamazepine is prescribed for treatment of 
A

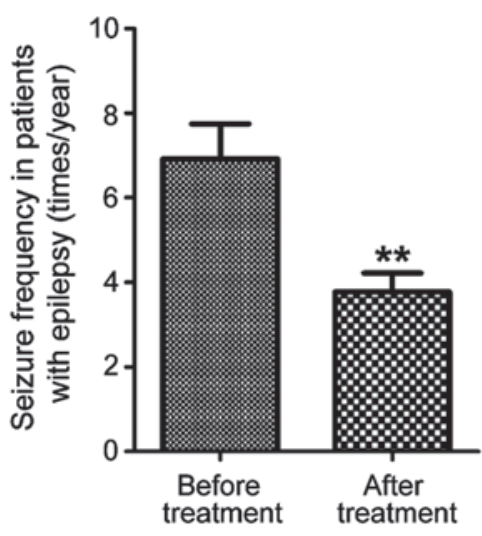

B

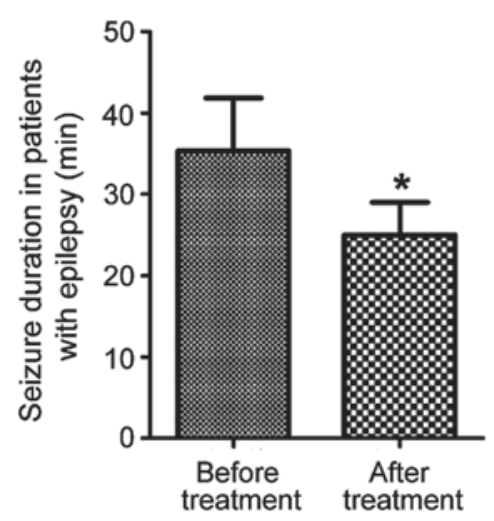

Figure 5. Seizure frequency and duration in patients with epilepsy. (A) Seizure frequency; (B) seizure duration ( $\left.\mathrm{P}<0.05,{ }^{* *} \mathrm{P}<0.01\right)$.

localized seizure and grand mal seizure (11). In clinic, traditional Chinese medicines were combined with carbamazepine to treat refractory epilepsy, which enhanced the efficacy of carbamazepine and improved the cure rate of refractory epilepsy (12). Kong et al (13) found that supplementation in epileptic patients with appropriate vitamin and other trace elements can effectively increase the efficacy of antiepileptic drugs and reduce drug resistance.

In this study, effects of carbamazepine combined with vitamin B12 on levels of plasma Hcy and serum hs-CRP and TNF- $\alpha$ in patients with epilepsy were investigated. It was found that carbamazepine combined with vitamin B12 can be used to treat a variety of epileptic seizures, and seizure frequency and duration were significantly reduced after 3 months of treatment. The results indicated that supplemental vitamin B12 had a synergistic effect with carbamazepine in treatment of epilepsy. In clinic, epileptic seizure was diagnosed using a double index evaluation based on EEG and observed clinical symptoms, which achieved high accuracy $(14,15)$. Ni et al reported that after carbamazepine was given intragastrically to rat epileptic model for one month, the plasma Hcy level was increased significantly (16), suggesting that oral administration of carbamazepine may increase the risk of elevated plasma Hcy levels. In this study, after 3 months of treatment of patients with epilepsy with carbamazepine combined with vitamin B12, the plasma Hcy level was significantly increased $(\mathrm{P}<0.01)$. Sidhu et al reported that supplementation of $\mathrm{B}$ vitamins can effectively reduce plasma levels of Hcy (17). However, this was debatable due to lack of supporting clinical data. The results from this study indicated that vitamin B12 did not reduce the level of Hcy. The elevated level of plasma Hcy due to antiepileptic drugs may lead to an increase in asymmetric dimethylarginine level. The latter is metabolized to become an endogenous inhibitor of nitric oxide (NO) synthase. Reduced NO is associated with cerebrovascular disease (18). It is recommended that patients with epilepsy should regularly check plasma level of Hcy during medications to avoid occurrence of adverse events. The effects of carbamazepine on levels of serum hs-CRP and TNF- $\alpha$ were detected by both ELISA and western blotting. The results showed that the levels of serum hs-CRP and TNF- $\alpha$ before and after treatment in patients with epilepsy were all higher than those in normal subjects. Whereas after treatment with carbamazepine combined with vitamin B12, these levels were significantly reduced compared to those before treatment. The results suggested that epilepsy mediated expression of hs-CRP and TNF- $\alpha$ in serum. Smeal et al reported that epilepsy may result in certain level of spontaneous damage to the body, releasing a variety of inflammatory factors (19). Thus, severity of seizures can be assessed by detecting the levels of inflammatory factors. Carbamazepine treatment of epilepsy reduced the damage caused by the disease, thereby reducing the expression of hs-CRP and TNF- $\alpha$ (20). The mechanism underlying carbamazepine modulation of levels of inflammatory factors is still unknown, which warrants further study.

In conclusion, carbamazepine combined with vitamin B12 can effectively treat a variety of seizures by reducing seizure frequency. Its antiepileptic efficacy may be associated with reduction of serum hs-CRP and TNF- $\alpha$ levels. However, carbamazepine poses a risk of increasing plasma Hcy level, which should be monitored in clinical application.

\section{Acknowledgements}

This study was supported by the Clinical Science and Technology Development Fund of Jiangsu University (no. JLY20160140) and the Xuzhou Science and Technology Planning Project (no. KC16SH025).

\section{References}

1. Peljto AL, Barker-Cummings C, Vasoli VM, Leibson CL, Hauser WA, Buchhalter JR and Ottman R: Familial risk of epilepsy: A population-based study. Brain 137: 795-805, 2014.

2. Brodie MJ, Besag F, Ettinger AB, Mula M, Gobbi G, Comai S, Aldenkamp AP and Steinhoff BJ: Epilepsy, antiepileptic drugs, and aggression: An Evidence-Based review. Pharmacol Rev 68: 563-602, 2016.

3. Kobau R, Cui W, Kadima N, Zack MM, Sajatovic M, Kaiboriboon K and Jobst B: Tracking psychosocial health in adults with epilepsy - estimates from the 2010 National Health Interview Survey. Epilepsy Behav 41: 66-73, 2014.

4. Cui W, Zack MM, Kobau R and Helmers SL: Health behaviors among people with epilepsy - results from the 2010 National Health Interview Survey. Epilepsy Behav 44: 121-126, 2015.

5. Yang RR, Wang WZ, Snape D, Chen G, Zhang L, Wu JZ, Baker GA, Zheng XY and Jacoby A: Stigma of people with epilepsy in China: Views of health professionals, teachers, employers, and community leaders. Epilepsy Behav 21: 261-266, 2011. 
6. Kaiboriboon K, Bakaki PM, Lhatoo SD and Koroukian S: Incidence and prevalence of treated epilepsy among poor health and low-income Americans. Neurology 80: 1942-1949, 2013.

7. Marino SE, Birnbaum AK, Leppik IE, Conway JM, Musib LC, Brundage RC, Ramsay RE, Pennell PB, White JR, Gross CR, et al: Steady-state carbamazepine pharmacokinetics following oral and stable-labeled intravenous administration in epilepsy patients: Effects of race and sex. Clin Pharmacol Ther 91: 483-488, 2012.

8. Hermann B, Seidenberg M, Sager M, Carlsson C, Gidal B, Sheth R, Rutecki P and Asthana S: Growing old with epilepsy: The neglected issue of cognitive and brain health in aging and elder persons with chronic epilepsy. Epilepsia 49: 731-740, 2008

9. Chen $\mathrm{H}, \mathrm{Xu} \mathrm{G}, \mathrm{Du} \mathrm{H}$, Yi M and Li C: Integrative analysis of gene expression associated with epilepsy in human epilepsy and animal models. Mol Med Rep 13: 4920-4926, 2016.

10. Berg AT, Baca CB, Loddenkemper T, Vickrey BG and Dlugos D Priorities in pediatric epilepsy research: Improving children's futures today. Neurology 81: 1166-1175, 2013.

11. Puranik YG, Birnbaum AK, Marino SE, Ahmed G, Cloyd JC, Remmel RP, Leppik IE and Lamba JK: Association of carbamazepine major metabolism and transport pathway gene polymorphisms and pharmacokinetics in patients with epilepsy. Pharmacogenomics 14: 35-45, 2013.

12. Doheny HC, Whittington MA, Jefferys JG and Patsalos PN: A comparison of the efficacy of carbamazepine and the novel antiepileptic drug levetiracetam in the tetanus toxin model of foca complex partial epilepsy. Br J Pharmacol 135: 1425-1434, 2002.

13. Kong ST, Lim SH, Lee WB, Kumar PK, Wang HY, Ng YL, Wong PS and Ho PC: Clinical validation and implications of dried blood spot sampling of carbamazepine, valproic acid and phenytoin in patients with epilepsy. PLoS One 9: e108190, 2014.

14. Zhou H, Wang N, Xu L, Huang HL and Yu CY: Clinical study on anti-epileptic drug with B vitamins for the treatment of epilepsy after stroke. Eur Rev Med Pharmacol Sci 21: 3327-3331, 2017.
15. Ahmed GF, Brundage RC, Marino SE, Cloyd JC, Leppik IE, Pennell PB, Ramsay RE and Birnbaum AK: Population pharmacokinetics of unbound and total drug concentrations following intravenously administered carbamazepine in elderly and younger adult patients with epilepsy. J Clin Pharmacol 53: 276-284, 2013.

16. Ni G, Qin J, Fang Z, Chen Y, Chen Z, Zhou J and Zhou L: Increased homocysteine levels in valproate-treated patients with epilepsy: A meta-analysis. BMJ Open 4: e004936, 2014.

17. Sidhu MK, Stretton J, Winston GP, Symms M, Thompson PJ, Koepp MJ and Duncan JS: Factors affecting reorganisation of memory encoding networks in temporal lobe epilepsy. Epilepsy Res 110: 1-9, 2015

18. Brady CB, Gaziano JM, Cxypoliski RA, Guarino PD, Kaufman JS, Warren SR, Hartigan P, Goldfarb DS and Jamison RL: Homocysteine lowering and cognition in CKD: The Veterans Affairs homocysteine study. Am J Kidney Dis 54: 440-449, 2009.

19. Smeal RM, Stewart KA, Iacob E, Fujinami RS, White HS and Wilcox KS: The activity within the CA3 excitatory network during Theiler's virus encephalitis is distinct from that observed during chronic epilepsy. J Neurovirol 18: 30-44, 2012.

20. Mbuba CK, Ngugi AK, Fegan G, Ibinda F, Muchohi SN, Nyundo C, Odhiambo R, Edwards T, Odermatt P, Carter JA, et al: Risk factors associated with the epilepsy treatment gap in Kilifi, Kenya: A cross-sectional study. Lancet Neurol 11: 688-696, 2012.

This work is licensed under a Creative Commons Attribution-NonCommercial-NoDerivatives 4.0 International (CC BY-NC-ND 4.0) License. 